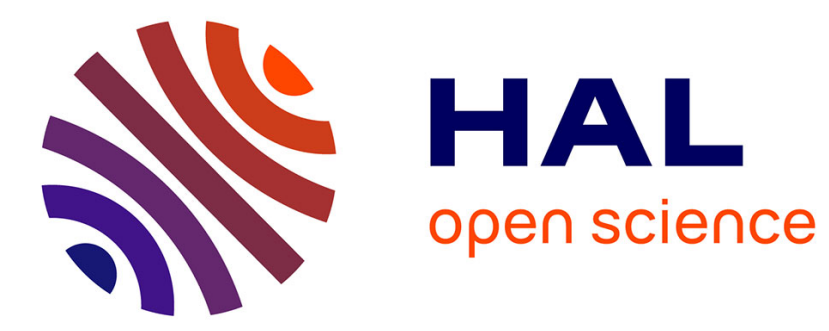

\title{
Comparisons of discrete and continuous propagators for the modelling of low tropospheric propagation
}

\author{
Hang Zhou, Alexandre Chabory, Rémi Douvenot
}

\section{To cite this version:}

Hang Zhou, Alexandre Chabory, Rémi Douvenot. Comparisons of discrete and continuous propagators for the modelling of low tropospheric propagation. EUCAP 2017 11th European Conference on Antennas and Propagation, Mar 2017, Paris, France. pp.ISBN : 978-1-5090-3742-1 10.23919/Eu-

CAP.2017.7928111 . hal-01527004

\section{HAL Id: hal-01527004 \\ https://hal-enac.archives-ouvertes.fr/hal-01527004}

Submitted on 28 May 2017

HAL is a multi-disciplinary open access archive for the deposit and dissemination of scientific research documents, whether they are published or not. The documents may come from teaching and research institutions in France or abroad, or from public or private research centers.
L'archive ouverte pluridisciplinaire HAL, est destinée au dépôt et à la diffusion de documents scientifiques de niveau recherche, publiés ou non, émanant des établissements d'enseignement et de recherche français ou étrangers, des laboratoires publics ou privés. 


\title{
Comparisons of Discrete and Continuous Propagators for the Modelling of Low Tropospheric Propagation
}

\author{
Hang Zhou ${ }^{12}$, Alexandre Chabory ${ }^{12}$, Rémi Douvenot ${ }^{12}$, \\ ${ }^{1}$ ENAC, TELECOM-EMA, F31055 Toulouse, France \\ ${ }^{2}$ Université de Toulouse, F-31400 Toulouse, France \\ e-mail:hang.zhou@ @echerche.enac.fr
}

\begin{abstract}
For modelling the propagation of electromagnetic waves in the low troposphere, the discrete mixed Fourier method is classically used. It is based on a finite-difference approximation of the ground boundary condition. However, the propagator of the split-step Fourier method is derived from continuous equations. In this paper, we apply the finite-difference approximation to the propagation equation. A discrete operator is derived from these discrete equations. The continuous and discrete propagators are compared. The accuracy of these propagators is numerically analysed in free space and over an impedance ground. We show that the discrete propagator avoids some numerical instabilities.
\end{abstract}

Index Terms-DMFT, propagation, discrete.

\section{INTRODUCTION}

To simulate the electromagnetic 2D wave propagation in the low troposphere, the parabolic equation is widely used [1]. This method is valid along a paraxial direction neglecting backward propagation. Hardin and Tappert have proposed an efficient numerical method based on the split-step Fourier methods [2]. In order to evaluate the field propagation iteratively at increasing distances, the computation is realized going back and forth from a spatial to a spectral representation.

The antenna pattern, the atmosphere condition, the ground composition, and the relief can be considered by these algorithms. To simulate the propagation over an impedance ground, the mixed Fourier transform method has been proposed by Dockery and Kuttler. For numerical applications, the discrete mixed Fourier transform (DMFT) is widely used [3]. Although it is based on a finite-difference approximation for the ground boundary condition, its propagator in the spectral domain is obtained from continuous equations.

In this paper, we derive a propagator in the spectral domain from discrete equations. The finite-difference approximation on the vertical axis is used for both the propagation equation and the boundary condition. Therefore, the solution is self consistent. Several tests are made in which simulation results are compared to analytic solutions. Free space propagation and propagation over several types of grounds are considered.

In the second section, the DMFT method is introduced. In the third section, the discrete propagator is derived from the discrete spectral wave equation. In the forth section, we compare the 2 propagators. A free-space propagation and propagation over impedance grounds are performed to test the accuracy.

\section{Discrete MiXed Fourier TRANSFORM}

In this section, we make a brief introduction of the discrete mixed Fourier transform (DMFT) method.

In the following, $u$ represents the classical reduced scalar field of the DMFT. The propagation is computed in the region $x \in$ $\left[0, x_{\max }\right]$ and $z \in\left[0, z_{\max }\right] . u$ is discretized on the vertical axis as $u_{m}=u(x, m \Delta z)$ for $m=0, \ldots, N_{z}$ and $\Delta z$ is the discretization step. The spectral representation of the DMFT is based on the finite-difference approximation of the Leontovich boundary condition along the vertical axis $z$

$$
\begin{aligned}
& w_{m}=\frac{u_{m+1}-u_{m-1}}{2 \Delta z}+\alpha u_{m}, \\
& \text { for } m=1, \ldots, N_{z}-1 .
\end{aligned}
$$

Its spectrum $W$ is obtained by applying a discrete sine transform (DST) to $w$. Finally, $W$ is also discretized as $W_{i}$ for $i=0, \ldots, N_{z}$.

In the spectral domain, the propagator $\Lambda$ given in the classical DMFT method is associated with the continuous propagation equation

$$
\begin{aligned}
& \left(\frac{\partial}{\partial x}+j\left(\sqrt{k^{2}-k_{z}^{2}}-k\right)\right) W_{i}=0, \\
& \text { for } i=1, \ldots, N_{z}-1,
\end{aligned}
$$

where $k$ is the wave number.

$W$ is propagated from range $x$ to $x+\Delta x$ using

$$
W_{i}(x+\Delta x)=\Lambda_{i} W_{i}(x), i=0, \ldots, N_{z},
$$

where

$$
\begin{aligned}
& \Lambda_{i}=\exp \left\{-j \Delta x\left(k_{x}-k\right)\right\}, \\
& \text { for } i=1, \ldots, N_{z}-1 .
\end{aligned}
$$

Furthermore, $\Delta x$ is the discretization step on $x$, and

$$
k_{x}= \begin{cases}\sqrt{k^{2}-\left(i \Delta k_{z}\right)^{2}} & \text { if } k^{2}-\left(i \Delta k_{z}\right)^{2} \geq 0, \\ -j \sqrt{\left(i \Delta k_{z}\right)^{2}-k^{2}} & \text { if } k^{2}-\left(i \Delta k_{z}\right)^{2}<0,\end{cases}
$$

where

$$
\Delta k_{z}=\pi / z_{\max } .
$$

In (3), the cases $i=0$ and $i=N_{z}$ can be assimilated to a ground wave and a sky wave, respectively. Their propagators are

$$
\begin{aligned}
& \Lambda_{0}=\exp \left\{-\frac{j \Delta x}{2 k \Delta z^{2}}(\log r)^{2}\right\}, \\
& \Lambda_{N_{z}}=\exp \left\{-\frac{j \Delta x}{2 k \Delta z^{2}}(\log (-r))^{2}\right\} .
\end{aligned}
$$




\section{SELF-CONSISTENT PROPAGATOR FOR DMFT}

In this section, we apply the finite-difference approximation on $z$ for both the propagation equation and the boundary condition equation.

The forward propagation equation under the finite-difference approximations on $z$ is

$$
\begin{aligned}
& \left(\frac{\partial}{\partial x}+j\left(\sqrt{k^{2}-p_{i}^{2}}-k\right)\right) W_{i}=0, \\
& \text { for } i=1, \ldots, N_{z}-1,
\end{aligned}
$$

where

$$
\begin{aligned}
& p_{i}=\frac{2}{\Delta z} \sin \left(\frac{\pi i}{2 N_{z}}\right), \\
& \text { for } i=1, \ldots, N_{z}-1 .
\end{aligned}
$$

The propagator is derived as

$$
\begin{aligned}
& \Lambda_{i}=\exp \left\{-j \Delta x\left(k_{x}-k\right)\right\}, \\
& \text { for } i=1, \ldots, N_{z}-1,
\end{aligned}
$$

with

$$
k_{x}= \begin{cases}\sqrt{k^{2}-p_{i}^{2}} & \text { if } k^{2}-p_{i}^{2} \geq 0 \\ -j \sqrt{p_{i}^{2}-k^{2}} & \text { if } k^{2}-p_{i}^{2}<0 .\end{cases}
$$

The ground and sky wave propagators are derived in a similar way. $W_{0}(x) r^{m}$ and $W_{N_{z}}(-r)^{N_{z}-m}$ already satisfy (8). Therefore, the propagators corresponding to the surface waves are

$$
\begin{aligned}
& \Lambda_{0}=\exp \left\{-j \Delta x\left(\sqrt{k^{2}+\frac{r+r^{-1}-2}{\Delta z^{2}}}-k\right)\right\}, \\
& \Lambda_{N_{z}}=\exp \left\{-j \Delta x\left(\sqrt{k^{2}+\frac{(-r)+(-r)^{-1}-2}{\Delta z^{2}}}-k\right)\right\} .
\end{aligned}
$$

\section{NUMERICAL TESTS}

\section{A. Comparison of the propagators}

Numerical tests are made to compare the continuous and discrete propagators.

The frequency is $300 \mathrm{MHz}$. The parameters of the simulation are $\Delta x=1 \mathrm{~m}, z_{\max }=10 \mathrm{~m}$, and $\Delta z=0.2 \mathrm{~m}$.

For the clarity of the figures, we only show 30 propagator values. The comparison of the propagators is shown in Fig 1. The blue crosses and red circles correspond to the expression of $\lambda$ in (4) and (10), respectively. The propagators located on the circle of radius 1.0 correspond to propagative modes, whereas the other ones correspond to evanescent modes. The norm and phase of the propagators are shown in Fig $1 \mathrm{~b}$ and 1c. We notice that, for the propagative modes of small order (approximately smaller than 20), the continuous and discrete propagators are very close. Differences become greater as the order increases.

Moreover, if we choose $\Delta z$ smaller, the difference between the 2 propagators becomes smaller. Indeed, in the discrete propagator, we apply the finite-difference approximation to the propagation equation. This approximation is closer to the exact expression when $\Delta z$ is chosen small.

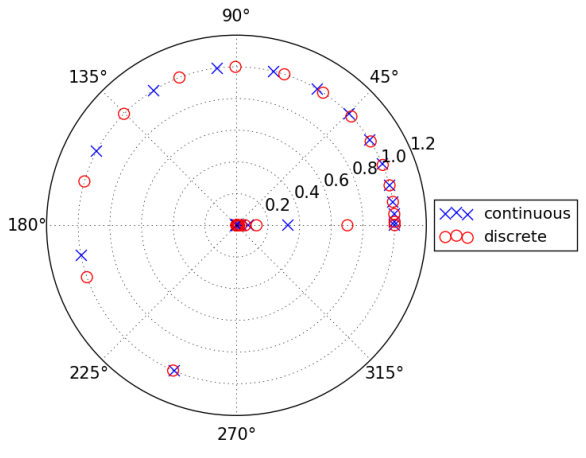

(a) Comparison of propagators

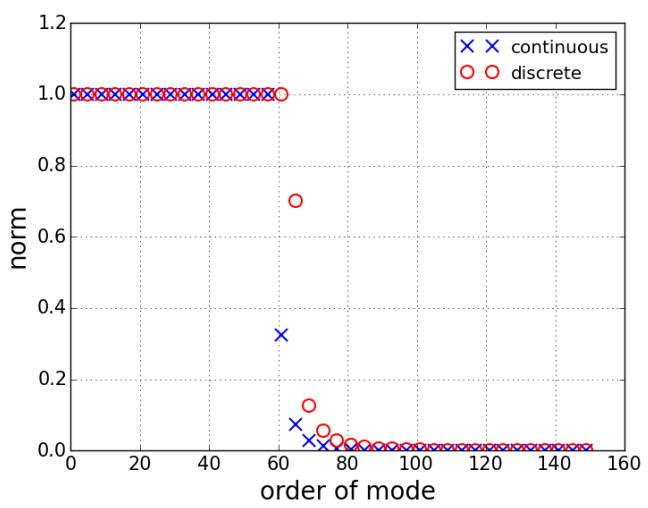

(b) Norm of propagators

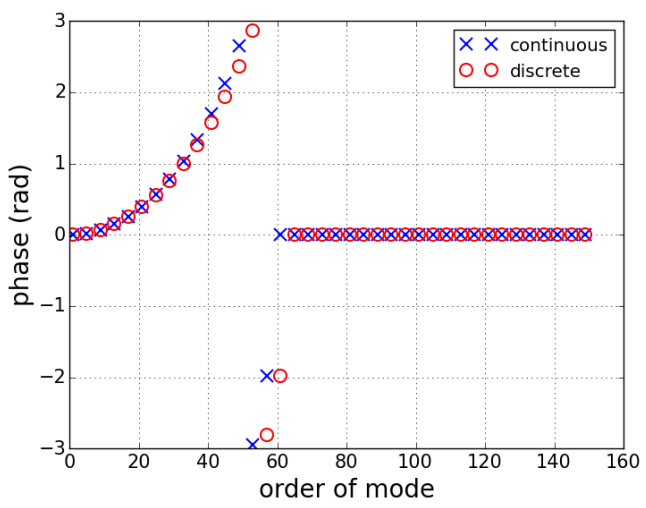

(c) Phase of propagators

Fig. 1. Comparison of the continuous and discrete propagators with $\Delta z=$ $0.1 \mathrm{~m}$

\section{B. Tests of propagations}

In this section, tests are made to compare the modelling of propagated electromagnetic field using the continuous and discrete propagators. A 2D complex source [4] is used as the initial electric field. The atmosphere is considered as homogeneous $(n=1)$. On the upper half area of the computation domain, we apply the Hanning window to make the apodization.

1) Free space propagation: $\mathrm{We}$ simulate the electromagnetic wave propagation in free space without reflection over the ground. We compare the simulation results 


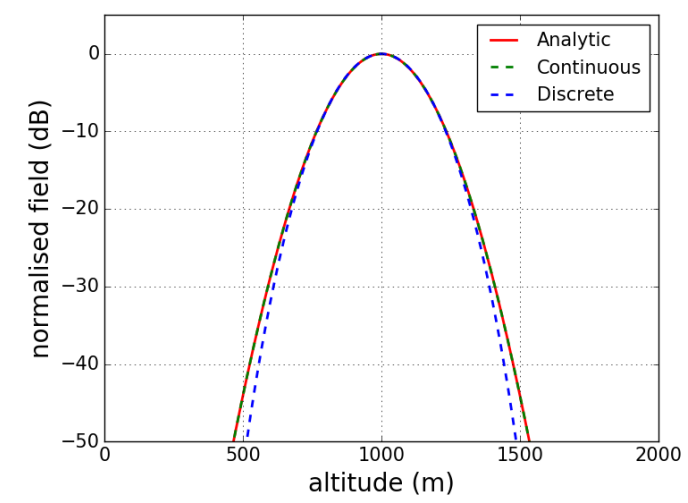

Fig. 2. Free space propagation of a $2 \mathrm{D}$ complex source at altitude $1000 \mathrm{~m}$ with $\Delta z=0.5 \mathrm{~m}$.

of the continuous and discrete propagators to the analytic solution of the complex source.

The parameters of the source are as follows: the frequency is $300 \mathrm{MHz}$, the waist size $W_{0}=3 \mathrm{~m}$, the $x$-position of the source center $x_{w 0}=-50 \mathrm{~m}$, the altitude of the source center is $1000 \mathrm{~m}$.

The parameters of the simulation are as follows: $x_{\max }$ is $2000 \mathrm{~m}, \Delta x$ is $100 \mathrm{~m} . z_{\max }$ is $4000 \mathrm{~m}$

First, We make a test with $\Delta z=0.5 \mathrm{~m}$. The final normalised fields are shown in Fig 2. Both the discretised and continuous propagator yield a good accuracy. Nevertheless, at lower and higher altitudes, the continuous one is more precise.

The reason is that an additional finite-difference approximation is applied to the propagation equation. This error is not negligible when $\Delta z$ is too large, especially for high-order modes.

2) Propagation over a dry ground: In this test, we simulate the propagation over a planar dielectric ground. We compare the simulation results of the continuous and discrete propagators to the 2-rays method.

The altitude of the source center is $20 \mathrm{~m}, x_{\max }$ is $5000 \mathrm{~m}$, and $\Delta z$ is $0.2 \mathrm{~m}$. The other parameters are the same as in the previous test. A dry ground is considered with $\epsilon_{r}=20$ and $\sigma=0.02 \mathrm{~S} / \mathrm{m}$

The final fields are shown in Fig 3. The simulation results are the same as the 2 rays method for both propagators.

3) Propagation over a very dry ground: We now choose a ground with a relative permittivity $\epsilon_{r}=2$ and a conductivity $\sigma=0.001 \mathrm{~S} / \mathrm{m}$. The other parameters are same as in the previous test.

The comparison of the final fields is shown in Fig 4. The field of the continuous case has small oscillations which are due to numerical instabilities. On the other hand, the discrete operator remains accurate here.

If the vertical step is chosen smaller, numerical instabilities can be so great that the simulated field becomes a numerical noise. The discrete formulation avoids these instabilities.

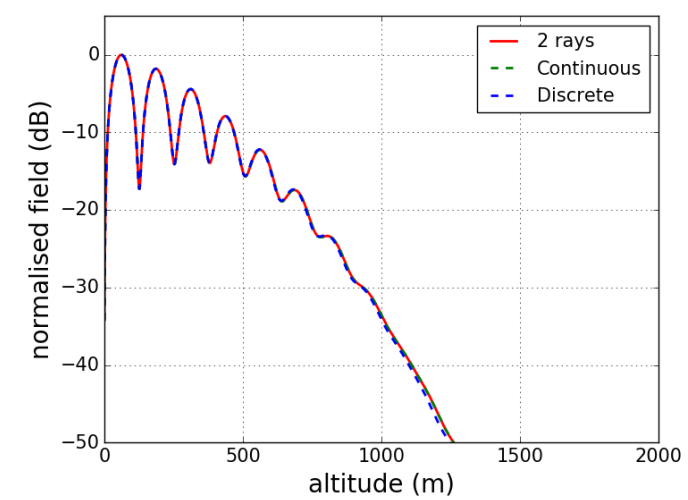

Fig. 3. Propagation of a 2D complex source at altitude $20 \mathrm{~m}$ over dry ground.

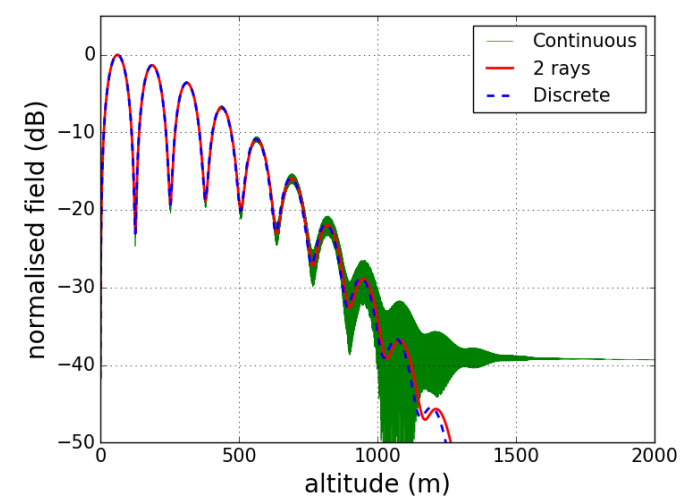

Fig. 4. Propagation of a $2 \mathrm{D}$ complex source at altitude $20 \mathrm{~m}$ over very dry ground.

\section{CONCLUSION}

In this paper, a discrete propagator for DMFT has been introduced. It has been derived from the discrete spectral propagation equation. Comparisons to the classical continuous propagator have been performed. The discrete propagator has the same precision as the continuous one when the vertical step is small. For some impedance ground conditions, the continuous operator is numerically unstable, whereas the discrete propagator remains accurate. It is believed that the discrete propagator is more self consistent and avoids numerical instabilities.

\section{REFERENCES}

[1] M. Levy, Parabolic Equation Methods for Electromagnetic Wave Propagation. IET, 2000, no. 45.

[2] R. H. Hardin and F. D. Tappert, "Applications of the split-step Fourier method to the numerical solution of nonlinear and variable coefficient wave equations," SIAM Review, vol. 15, no. 1, pp. 423-429, 1973.

[3] G. D. Dockery and J. R. Kuttler, "An improved impedance-boundary algorithm for Fourier split-step solutions of the parabolic wave equation," IEEE Transactions on Antennas and Propagation, vol. 44, no. 12, pp. 1592-1599, 1996.

[4] G. A. Deschamps, "Gaussian beam as a bundle of complex rays," Electronics Letters, vol. 7, no. 23, pp. 684-685, November 1971. 